\title{
What is top-down about contingent capture?
}

\author{
Artem V. Belopolsky, Daniel SchreiJ, And Jan Theeuwes \\ Vrije Universiteit Amsterdam, Amsterdam, The Netherlands
}

\begin{abstract}
In the present study, we explored the mechanisms involved in the contingent capture phenomenon, using a variant of the classic precuing paradigm of Folk, Remington, and Johnston (1992). Rather than keeping the target fixed over a whole block of trials (as has traditionally been done with contingent capture experiments), we encouraged participants to adopt a top-down set before each trial. If top-down attentional set determines which property captures attention, as is claimed by the contingent capture hypothesis, one would expect that only properties that match the top-down set would capture attention. We showed that even though participants knew what the target would be on the upcoming trial, both relevant and irrelevant properties captured attention (Experiment 1). An intertrial analysis (Experiments 1 and 2) showed that previous contingent capture findings may, to a large extent, be explained by intertrial priming. In addition, when participants were further forced into adopting the required top-down set (Experiments 3 and 4), irrelevant cues were suppressed, suggesting that top-down control might operate through disengagement of attention from the location of a property that does not match top-down goals. The present findings suggest that top-down control and intertrial priming make their own distinct contributions to the contingent capture phenomenon.
\end{abstract}

Since the early 1990s, there has been a considerable debate regarding the extent to which the allocation of spatial attention is under top-down or bottom-up control. On the one hand, it has been argued that the allocation of attention is initiated voluntarily in line with behavioral goals (Folk, Remington, \& Johnston, 1992). Alternatively, it has been claimed that events that are salient enough are selected regardless of the current top-down set (Theeuwes, 1992, 1994). In the latter case, when objects receive priority of processing independently of the volitional goals of the observer, one refers to this as attentional capture. When such an object not only captures attention, but also triggers an exogenous saccade to the location of the event, this is referred to as oculomotor capture (Theeuwes, Kramer, Hahn, Irwin, \& Zelinsky, 1999).

Evidence for bottom-up attentional capture comes from the irrelevant singleton paradigm developed by Theeuwes (1991, 1992, 1994). In this task, participants search for one particular feature singleton (the target) while, on some trials, another irrelevant salient singleton (the distractor) is also present. Critically, the presence of the irrelevant singleton increases the time needed to find the relevant singleton. Reaction time (RT) cost led Theeuwes $(1991,1992)$ to conclude that the irrelevant salient singleton captured attention automatically. On the basis of these findings, Theeuwes (1991, 1992; see also Hickey, McDonald, \& Theeuwes, 2006; Schreij, Owens, \& Theeuwes, 2008; Theeuwes, 2004) argued that attentional capture is basically bottom up and is not affected by a volitional top-down attentional set.

In a recent study, Theeuwes and Van der Burg (2008) explicitly addressed the question of whether volitional top-down set can affect attentional capture in a variant of the additional-singleton paradigm in which the exact target was unknown (i.e., the target was either a diamond among circles or a circle among diamonds). On each trial, an instructional cue (the word diamond or circle) indicated with $100 \%$ validity the identity of the upcoming target. For example, when the word diamond was presented, the participants knew that the target singleton on the upcoming trial would be a diamond. The results showed that the instructional cue was effective in reducing RT, indicating that the participants actively used the cue. However, at the same time, this top-down set could only reduce but not eliminate the distracting effect of the irrelevant color singleton in the additional-singleton paradigm.

The idea that selection is completely under volitional top-down control comes from a different paradigm known as the spatial precuing paradigm, developed by Folk and colleagues (Folk et al., 1992; Folk, Remington, \& Wright, 1994). In this paradigm, the search display is preceded by a cue display. In Folk et al.'s classic 1992 study, the target display consisted of either a color or an onset singleton, and observers were required to identify the unique element. In the color display, the target was red, and the other three elements were white. In the onset display, only one element was presented, and so the target was characterized as being the only element presented with an abrupt onset. Immediately preceding the target display at a 150 -msec stimulus onset asynchrony (SOA), a cue display was presented: This cue display consisted of either a color cue (in which one location was surrounded by red dots and the other three locations were surrounded by white dots) or an onset cue

A.V. Belopolsky, a.belopolsky@psy.vu.nl 
(in which one location was surrounded by an abrupt onset of white dots and the remaining locations remained empty). All conditions were factorially combined, and the target type remained constant within block of trials.

The critical finding of Folk et al.'s (1992; Folk et al., 1994) studies was that only when the search display was preceded by a to-be-ignored featural singleton (the cue) that matched the singleton for which observers were searching did the cue capture attention. Thus, when searching for a red target singleton, attention automatically shifted to the location of the irrelevant red cue that preceded the search display, whereas the irrelevant onset had no effect on performance. The result suggests that the top-down attentional set determines the selection priority. When observers are set for a particular feature singleton, only elements that match this top-down attentional set will capture attention. Feature singletons that do not match top-down attentional sets will simply be ignored. "With a control setting established, events exhibiting the critical properties will involuntarily summon attention, whether or not the event is actually relevant to task performance. Stimuli not exhibiting these properties will not involuntarily summon attention" (Folk et al., 1992, p. 1041). The underlying theoretical notion of the contingent capture hypothesis proposed by Folk and colleagues is that capture is fully contingent on the top-down set adopted by the observer and salient objects with irrelevant properties are simply filtered out (Folk \& Remington, 1998). On the other hand, finding attentional capture by an event that does not exhibit target-related properties while the appropriate attentional set has been established would constitute a violation of the strong version of the contingent capture hypothesis. The contingent capture hypothesis appears to be the generally accepted way of accounting for top-down control in visual search (see recent reviews by Burnham, 2007; Rauschenberger, 2003; but see Schreij et al., 2008; Theeuwes, 2004).

The present study was designed to explore the mechanisms involved in contingent capture and pursued two goals. First of all, since, in the classic precuing paradigm, the target remains the same for a whole block of trials (e.g., search for a color singleton), it leaves the possibility that such fixed attentional set for the target feature was not truly top down, but instead driven by automatic intertrial priming (Maljkovic \& Nakayama, 1994). This was examined in Experiments 1 and 2, in which we mixed the trials with onset and color targets within a block, preventing the participants from adopting a top-down set for a specific feature. If intertrial priming can explain contingent capture, we would expect the same contingent capture pattern of results here: Only the cues that match the target on the previous trial should capture attention.

The second goal of the present study was to test whether a top-down set is capable of guiding attention in a contingent fashion on a trial-by-trial basis. As in Theeuwes and Van der Burg (2008), we presented an instructional cue at the beginning of each trial indicating with $100 \%$ validity the target that would be presented on the upcoming trial (Experiment 1). In Experiment 3, the cue indicated which target, onset or color, the participants had to search for and respond to. They had to withhold their response if a differ- ent target was presented. In Experiment 4, we even let the participants choose for themselves which target dimension they wanted to search for and respond to. We employed a variant of the classic precuing paradigm of Folk et al. (1992), which allowed us to determine whether the precue captured spatial attention or not. If visual selection is truly dependent on volitional top-down set, we would expect basically the same results as those reported by Folk et al. (1992): Only features that matched the current attentional set should capture attention. However, if it turns out to be impossible to prepare for a specific target on the upcoming trial in a top-down way, one would expect not only that the singleton that matched the top-down set would capture attention, but also that the feature singleton that did not match the top-down set would capture attention.

\section{EXPERIMENT 1}

In Experiment 1, we examined the possibility of creating a top-down set for a specific target feature on a trial-by-trial basis. The task used was basically that of the classic precuing paradigm of Folk et al. (1992), in which participants had to search for either a color or an onset singleton. The search display was immediately preceded by a nonpredictive color or onset cue. Every trial began with an instructional cue (RED or WHITE), which, with $100 \%$ validity, indicated the feature of the upcoming target (see Figure 1). Using the word cue was crucial to our goal of inducing a top-down set, since it ensured that a top-down set was not contaminated by any bottom-up information about the upcoming feature (Theeuwes, Reimann, \& Mortier, 2006; Theeuwes \& Van der Burg, 2008). This condition was compared with a condition in which a neutral cue (NEUTRAL) was provided. If a top-down set created on every trial could influence selection, only the cues that match this set would produce a cuing effect. However, if such a top-down set could not modulate selection, all types of cues, even the ones that do not match the topdown set, would produce cuing effects.

\section{Method}

Participants. Eighteen students ( 6 of them male; mean age $=$ 21 years; range, $18-29$ years) participated in the experiment as paid volunteers or in exchange for course credit. One participant was replaced because of an excessive error rate $(>12 \%)$.

Stimuli. A trial started with the presentation of a white $\left(60.9 \mathrm{~cd} \cdot \mathrm{m}^{-2}\right)$ instructional cue (RED, WHITE, or NEUTRAL; Courier New, font size 18) at the center of a black display for $500 \mathrm{msec}$ (see Figure 1). It was followed by a fixation display, which consisted of a white fixation cross, surrounded above, below, left, and right at a $7.36^{\circ}$ distance by four white (CIE: $.277, .298 ; 45.75 \mathrm{~cd} \cdot \mathrm{m}^{-2}$ ) boxes $\left(3.68^{\circ} \times 3.68^{\circ}\right)$. After $500 \mathrm{msec}$, one of the boxes was cued by either an onset or a color cue for $50 \mathrm{msec}$. The onset cue consisted of four small white circles $\left(0.3^{\circ}\right)$ presented around the four sides of the cued box. The color cue consisted of the same four small circles presented around all four boxes; however, the circles around the cued box were colored red (CIE: .628, .343; $9.61 \mathrm{~cd} \cdot \mathrm{m}^{-2}$ ), whereas the circles surrounding the other boxes were colored white. The cue display was replaced by a fixation display for another $100 \mathrm{msec}$, followed by either an onset or a color target display for $50 \mathrm{msec}$. The onset target was a single white character " $\mathrm{X}$ " or " $=$," presented in one of the boxes. The color target was a red "X" or " $=$ " presented in one of the boxes, together with white distractor characters (“\%," "\#," and " \&"; 



Onset Cue

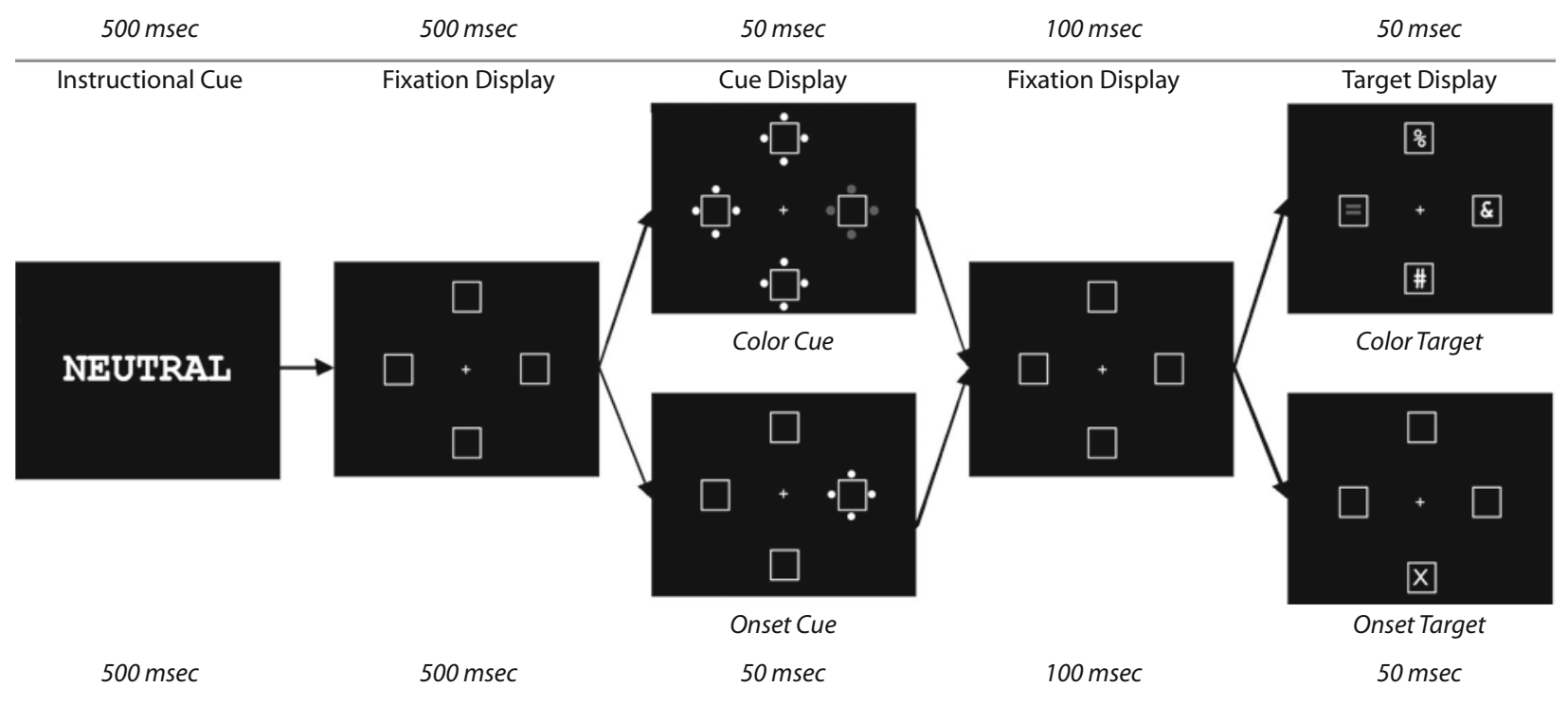

Figure 1. Example of trials in Experiment 1. Dark gray represents the red color. The instructional cue was $100 \%$ valid in indicating the target on the upcoming trial. Participants made a speeded response to the target shape ("X" or "="). 
all characters, $2.9^{\circ} \times 2.9^{\circ}$ ) that were presented in the other boxes. The intertrial interval was $500 \mathrm{msec}$.

Design and Procedure. All the conditions were within subjects and were randomly mixed within blocks. In the informative instructional cue condition, the participants received either an instructional cue RED, which with $100 \%$ validity informed them that the color target would be presented, or an instructional cue WHITE, which with $100 \%$ validity informed them that the onset target would be presented. In these conditions, the participants had every opportunity to adopt a top-down set for a specific target feature and were instructed about the advantage of doing that. In the uninformative instructional cue condition, the participants received the instructional cue NEUTRAL, which informed them only that both onset and color targets were equally likely to be presented.

In all the conditions, an onset or a color cue was equally likely to precede the target display. As in the classic Folk et al. (1992) study, the cue predicted the target location at a chance level, meaning that the target appeared at the cued location on $25 \%$ of the trials (valid cue) and appeared at one of the uncued locations on $75 \%$ of the trials (invalid cue).

The participants received 10 experimental blocks of 96 trials each, preceded by 1 practice block (50 trials). In each experimental block, the uninformative cue condition occurred on half of the trials, and on the other half of the trials, the color- and onset-informative instructional cues were equally likely to occur. The participants were instructed to respond quickly and accurately by pressing the " $\mathrm{x}$ " key when the target was " $\mathrm{X}$ " and the " $\mathrm{m}$ " key when the target was " =." After each block, the participants received feedback about their performance.

\section{Results}

Trials on which the participants responded more quickly than $150 \mathrm{msec}$ or more slowly than $1,100 \mathrm{msec}$ were excluded from further analysis. This led to a loss of $0.8 \%$ of the trials.

Mean correct RTs for the trials with informative and uninformative instructional cues are presented in Figures 2 and 3, respectively. A within-subjects ANOVA with instructional cue (informative cue vs. uninformative), target property (color vs. onset), cue property (color vs. onset), and cue validity (valid vs. invalid) as factors was performed on the mean correct RTs. The analysis revealed a main effect of the informative cue $[F(1,17)=8.85, p<$ $.01]$, indicating that the participants responded more quickly to a target when they were informed about the feature of the upcoming target $(466 \mathrm{msec})$ than when they were not $(474 \mathrm{msec})$. This indicates that the participants were successful in adopting the appropriate top-down set for the upcoming target.

To investigate whether the adopted top-down set influenced attentional capture, the data for the informative instructional cues were submitted to a $2 \times 2 \times 2$ ANOVA with target property (color vs. onset), cue property (color vs. onset), and cue validity (valid vs. invalid) as factors. For the informative instructional cue condition, mean correct RTs for valid and invalid trials at each combination of cue and target property are presented in Figure 2. There was a main effect of target property $[F(1,17)=$ 24.47, $p<.001]$, suggesting that the participants were faster in responding to the onset targets than to the color targets. There was a significant interaction of target and cue properties $[F(1,17)=10.80, p<.005]$, indicating that for the color targets, trials with color cues were,

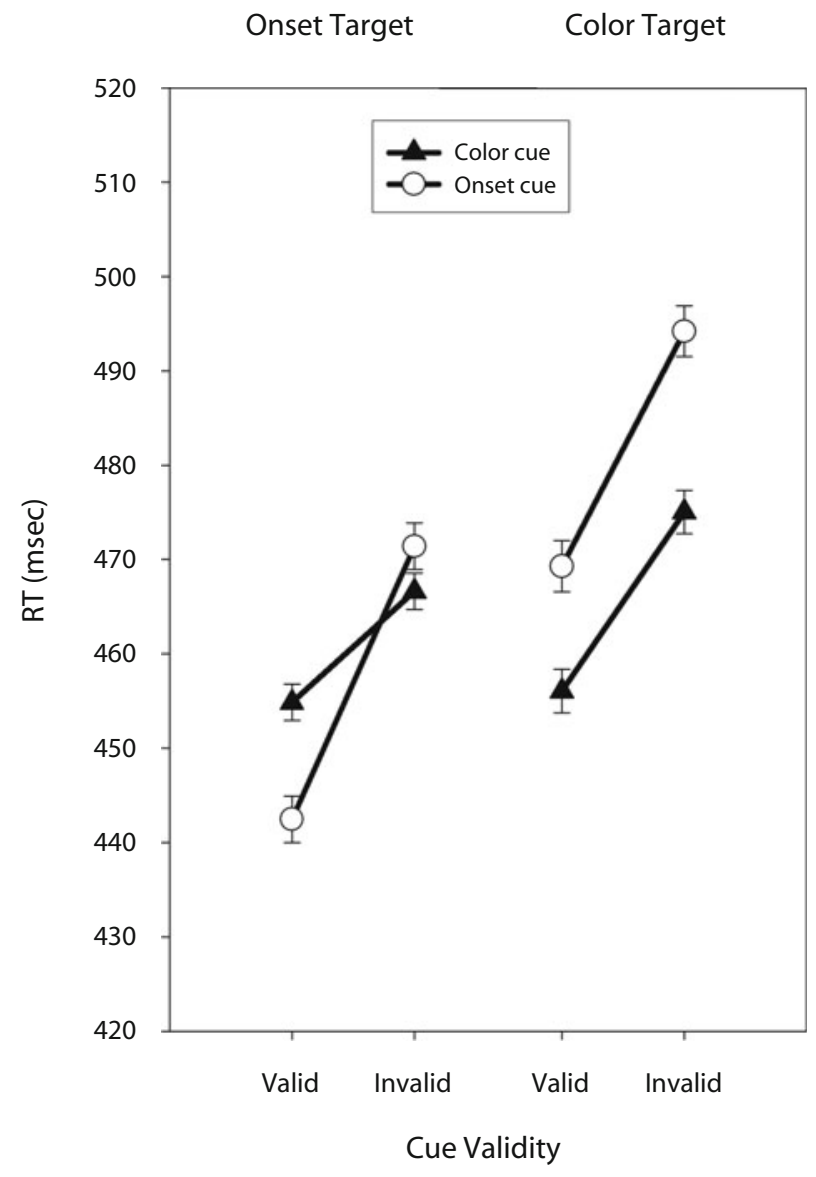

Figure 2. Mean reaction times (RTs) for the informative instructional cues as a function of target property, cue property, and cue validity in Experiment 1. The error bars represent standard errors of the means for within-subjects designs normalized for the cue validity factor.

overall, responded to slightly more quickly than were trials with onset cues, which was not the case for the onset targets. RTs for the invalid cues were significantly longer than those for the valid cues $[F(1,17)=44.29$, $p<.001]$. The analysis also showed a main effect of cue property $[F(1,17)=5.95, p<.05]$, with onset cues resulting, overall, in slower responses than did color cues. In addition, cue validity interacted with cue property $[F(1,17)=7.48, p<.05]$, indicating that the overall effect of cue validity was greater for the onset cues than for the color cues.

Importantly, the three-way interaction between target property, cue property, and cue validity failed to reach significance $[F(1,17)=1.80, p=.20]$. The absence of this interaction suggests that the top-down set did not prevent capture by a nonmatching cue property: Regardless of top-down set adopted, both cues captured attention (see Figure 2).

The influence of cue property on cue validity was further explored in planned comparisons conducted at each level of target property. For the color targets, there was a main effect of cue property $[F(1,17)=36.17, p<.001]$ 
and cue validity $[F(1,17)=26.81, p<.001]$. Importantly, the interaction between cue property and cue validity was not significant $[F(1,17)=1.23, p=.28]$. The absence of the interaction suggests that even though the participants adopted a top-down set for color, both color and onset cues resulted in a similar validity effect (19 and $25 \mathrm{msec}$, respectively; see Figure 2, right side).

For the onset targets, there was only a main effect of cue validity $[F(1,17)=45.67, p<.001]$. The interaction between cue property and cue validity was significant $[F(1,17)=7.02, p<.05]$, suggesting that onset and color cues captured attention to a different extent. Although the capture by onset cues $(29 \mathrm{msec})$ was larger than the capture by color cues $(12 \mathrm{msec})$, post hoc tests showed that both color cues $[t(17)=3.01, p<.01]$ and onset cues $[t(17)=5.89, p<.001]$ produced a significant effect of cue validity (Figure 2, left side). Just as the top-down set for color targets could not prevent capture by the onset cues, a top-down set for onsets could not prevent color cues from capturing attention.

Error rates were relatively low (7.3\%), and the ANOVAs revealed no significant effects or interactions.

Intertrial effects in the uninformative instructional cue condition. The uninformative instructional cue (neutral) was ambiguous with regard to the feature of the upcoming target, meaning that the participants could not adopt an appropriate top-down set. To determine whether, in this condition, on a given trial the participants were driven by the target detected and processed on the previous trial, we sorted the trials on the basis of whether the target on the previous trial was a color target or an onset target. Mean correct RTs for valid and invalid trials at each combination of cue property, the property of the target on the previous trial are presented in Figure 3. The data were analyzed using a within-subjects ANOVA with previous target property (color vs. onset), current target property (color vs. onset), cue property (color vs. onset), and cue validity (valid vs. invalid) as factors. There was a main effect of current target property $[F(1,17)=16.68$, $p<.005]$, indicating that, overall, onset targets were detected more quickly than color targets, and a main effect of cue validity $[F(1,17)=17.22, p<.005]$. As before, cue validity interacted with cue property $[F(1,17)=5.36, p<$ $.05]$, indicating that, overall, the effect of cue validity was greater for the onset cues than for the color cues. There was a significant interaction between previous target property and current target property $[F(1,17)=5.21, p<$ $.05]$, suggesting that responses were faster when the target property was repeated (a basic priming effect; Maljkovic \& Nakayama, 1994). Importantly, there was a significant three-way interaction among previous target property, cue property, and cue validity $[F(1,17)=7.42, p<.05]$, suggesting that the cue validity effect varied as a function of both cue property on the current trial and target property on the previous trial (Figure 3). No other main effects or interactions were significant.

This three-way interaction was further explored in planned comparisons conducted at each level of the previous target property. When the target on the previous trial was an onset target, there was a main effect of cue validity

\section{Uninformative Cue}

Previous Onset Target Previous Color Target

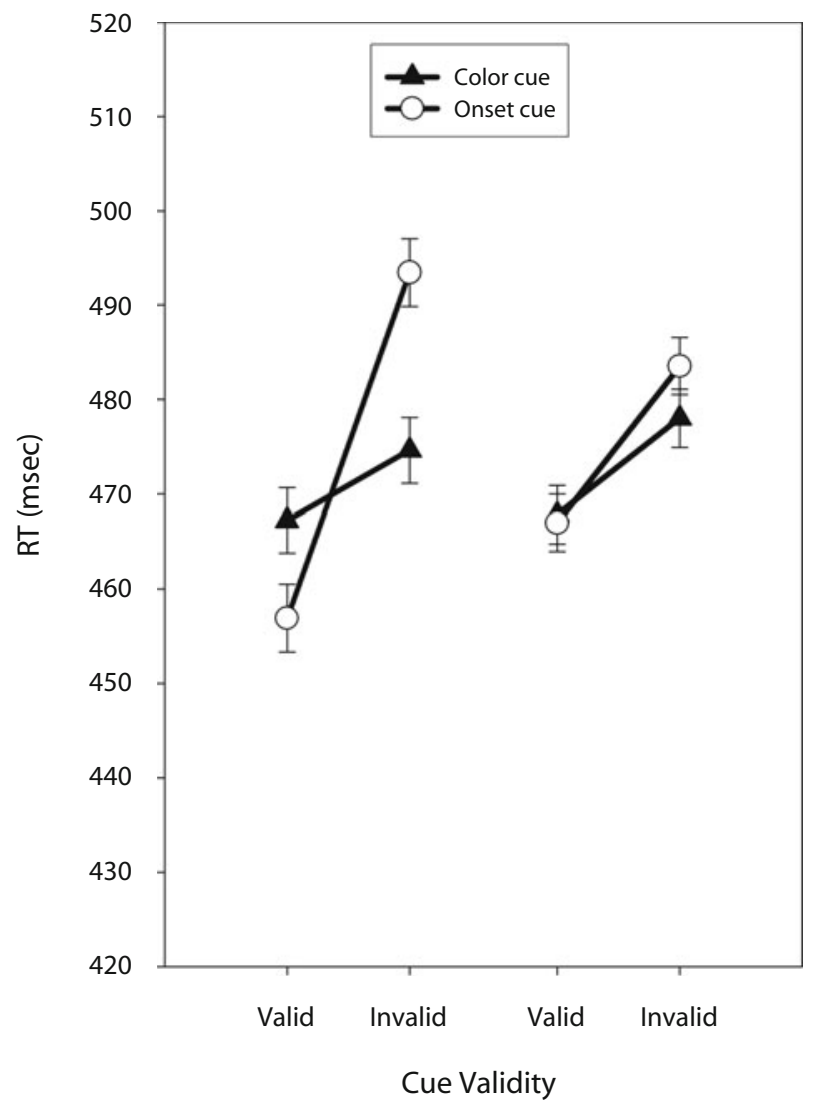

Figure 3. Mean reaction times (RTs) (neutral top-down set) for the uninformative instructional cues as a function of target property on the previous trial, cue property, and cue validity in Experiment 1. The error bars represent standard errors of the means for within-subjects designs normalized for the cue validity factor.

$[F(1,17)=20.61, p<.001]$ and a significant interaction between cue validity and cue property $[F(1,17)=10.75$, $p<.005]$. Post hoc tests showed that the effect of cue validity was present only for the onset cues $[F(1,17)=$ $27.11, p<.001]$ and not for the color cues $(F<1)$. When the target on the previous trial was a color target, there was a main effect of cue validity $[F(1,17)=6.86, p<.05]$, but the interaction between cue property and cue validity was not significant $(F<1)$, indicating that both cues captured attention to a similar extent. The results suggest that in the absence of a top-down set, the target on the previous trial determines which cue property captures attention on the following trial.

\section{Discussion}

The results of Experiment 1 clearly demonstrate a limitation of the contingent capture hypothesis. Although the participants were given ample opportunity to adopt a specific top-down set on every trial, this could not prevent attentional capture by the cues that were not part of the top-down set. In the case of the top-down set for color 
targets, the participants were captured equally strongly by both color and onset cues. Therefore, this top-down set had no effect on attentional capture. In the case of the top-down set for onset targets, the participants were captured more strongly by the onset cues than by the color cues, which indicates that top-down information was able to bias attentional capture. It is crucial, however, that the color cues still captured attention, although they were not a part of the current top-down set.

The analysis of intertrial effects indicates that in the absence of the top-down set, the target on the previous trial is an important factor in determining which cue property will capture attention on the current trial. Note that such intertrial effects are considered to be a form of bottom-up priming (Maljkovic \& Nakayama, 1994). When the target on the previous trial was an onset, only onset cues captured attention on the following trial, while the color cues were ignored. When the target on the previous trial was a color target, the color cues captured attention to the same extent as the onset cues. This asymmetry suggests that it is much more difficult to ignore the onset cues (e.g., Jonides \& Yantis, 1988; Schreij et al., 2008; Theeuwes, 1990, 1994, 1995; Theeuwes, Kramer, Hahn, \& Irwin, 1998; Yantis \& Jonides, 1984). However, whereas the top-down set was never able to produce exclusive control over attentional selection, the intertrial priming was able to deliver this kind of control, at least for the onset targets.

One potential concern about our intertrial priming results is that the previous trials used in this analysis included a mixture of trials with informative cues and trials with uninformative cues. Therefore, it is possible that, at least partially, the effect of the previous trial on attentional capture was due to the perseverance of attentional set established on the previous trial (using the informative cue), instead of mere processing of the target on the previous trial. In order to address this issue, in Experiment 2, we did not provide any information about the upcoming target (similar to the uninformative cues in Experiment 1). If the results in Experiment 1 were, in fact, due to bottom-up intertrial priming, we would expect to replicate the modulation of attentional capture by the target on the previous trial in Experiment 2.

\section{EXPERIMENT 2}

In Experiment 2, participants were asked to search either for an onset target or for a color target. They were not informed about the upcoming target and, therefore, could not establish a top-down set for a specific target property. The goal of this experiment was to examine whether the target selected on the previous trial would be able to determine what features captured attention on the current trial.

\section{Method}

Participants. Thirteen students ( 5 of them female; mean age $=$ 21 years; range, $18-25$ years) participated in the experiment as paid volunteers or in exchange for course credit. One participant was replaced because of an excessive error rate $(>11 \%)$.

Stimuli, Design, and Procedure. The experiment was very similar to Experiment 1, except that no instruction cues were presented. The trials with color and onset targets were randomly mixed within the blocks, and the participants did not know what type of target to expect. The trial started with a presentation of a fixation display for a random duration of 1,000-1,400 msec. It was followed by a 100 -msec offset of the fixation cross, and then the fixation display reappeared for another $100 \mathrm{msec}$. This was followed by one of the boxes being cued by either an onset or a color cue for $50 \mathrm{msec}$. The cue display was replaced by a fixation display for another $100 \mathrm{msec}$, followed by either an onset or a color target display for $50 \mathrm{msec}$.

The participants received 16 experimental blocks of 64 trials each, preceded by 1 practice block ( 50 trials). The participants were instructed to respond quickly and accurately by pressing the " $\mathrm{x}$ " key when the target was " $X$ " and the " $\mathrm{m}$ " key when the target was " =." After each block, the participants received feedback about their performance. The rest of the experiment was identical to Experiment 1.

\section{Results}

Trials on which the participants responded more quickly than $150 \mathrm{msec}$ or more slowly than $800 \mathrm{msec}$ were excluded from further analysis. This led to a loss of $1 \%$ of the trials.

Mean correct RTs for valid and invalid trials at each combination of cue property and target property on the previous trial are presented in Figure 4. To investigate whether the property of the target on the previous trial influenced which features captured attention on the current trial, mean correct RTs were submitted to a within-subjects ANOVA with previous target property (color vs. onset), current target property (color vs. onset), cue property (color vs. onset), and cue validity (valid vs. invalid) as factors.

There was a main effect of current target property $[F(1,12)=11.59, p<.01]$, indicating that, overall, onset targets were detected more quickly than color targets, and a main effect of cue validity $[F(1,12)=43.02, p<.001]$. There was also a significant interaction between current target property and cue property $[F(1,12)=7.19, p<$ $.05]$, suggesting that for the color targets, the participants responded slightly more quickly when the cue and current target properties matched but responded more slowly to the onset targets when there was a matching onset cue. This indicates that matching cues caused some interference, at least for the onset targets. Confirming this observation, a three-way interaction among current target property, cue property, and validity $[F(1,12)=12.38$, $p<.005]$ was also significant. For both onset and color targets, the match between the cue property and the current target property led to a reduced validity effect (see Table 1). Importantly, the three-way interaction among previous target property, cue property, and cue validity was significant $[F(1,12)=6.90, p<.05]$. This interaction suggests that the target property on the previous trial influenced which cue property captured attention on the current trial. The pattern of this interaction is very similar to the typical contingent capture result and suggests that in the absence of a top-down set, the target property on the previous trial affects which cue property captures attention on the current trial (see Figure 4). No other main effects and interactions were significant.

This three-way interaction was further explored in planned comparisons conducted at each level of the previous target property. When the target on the previous trial was an onset target, there was a significant interaction be- 
Previous Onset Target Previous Color Target

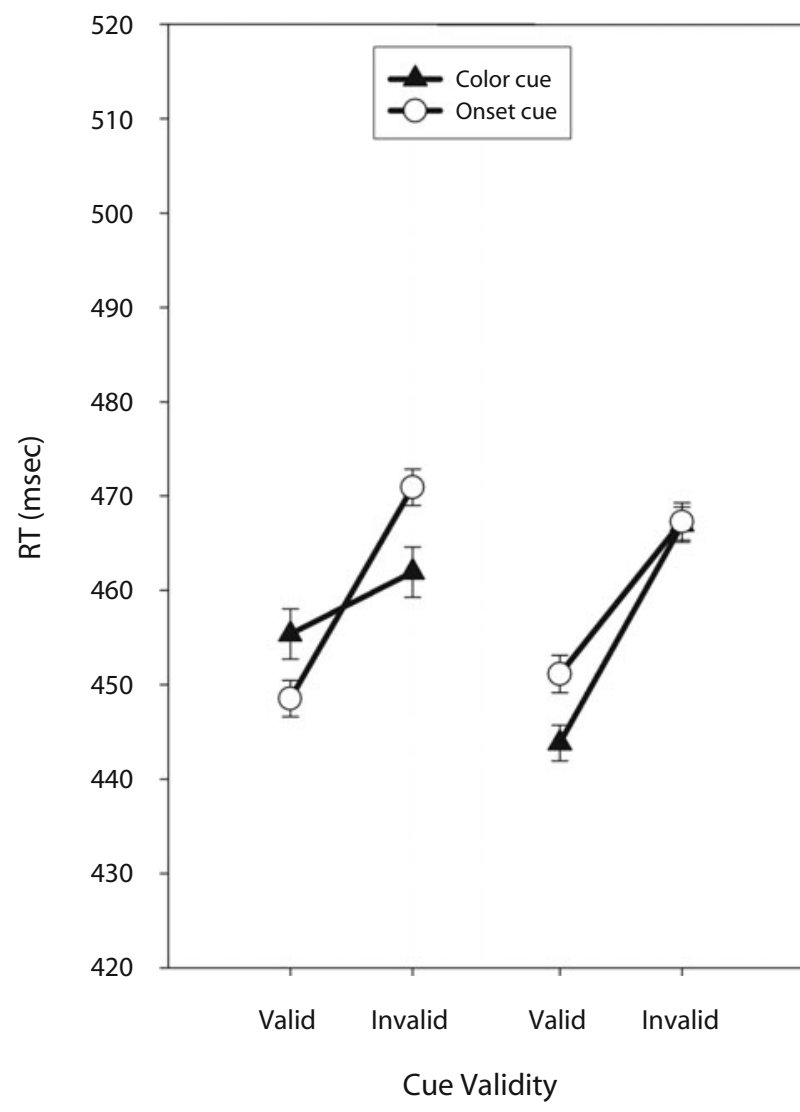

Figure 4. Mean reaction times (RTs) as a function of target property, cue property, and cue validity in Experiment 2. The error bars represent standard errors of the means for withinsubjects designs normalized for the cue validity factor.

tween cue validity and cue property $[F(1,12)=5.55, p<$ $.05]$. Post hoc tests showed that the effect of cue validity was present only for the onset cues [22 msec; $F(1,12)=33.58$, $p<.001]$ and not for the color cues $[7 \mathrm{msec} ; F(1,12)=$ $1.54, p=.24]$. When the target on the previous trial was a color target, the interaction between cue property and cue validity did not reach significance $[F(1,12)=1.78$, $p=.21]$, indicating that both onset $(16 \mathrm{msec})$ and color $(23 \mathrm{msec})$ cues captured attention to a similar extent.

An ANOVA on the error rates revealed a marginal main effect of previous target property $[F(1,12)=4.64, p=$ $.052]$, suggesting that the participants were slightly more accurate when the previous target was a color singleton. There was a main effect of cue validity $[F(1,12)=6.23$, $p<.05$ ], with participants being more accurate when the target was validly cued. There was also a significant interaction between current target property and cue property $[F(1,12)=10.07, p<.01]$, reflecting a masking phenomenon already observed in RTs. The participants were less accurate in responding to the targets that matched the cue property (see Table 1). There was also a significant twoway interaction between target property on the previous trial and cue validity $[F(1,12)=4.99, p<.05]$, suggest- ing that validity effect was larger when the target was a color singleton on the previous trial than when it was an onset. The three-way interaction among previous target property, cue property, and cue validity did not reach significance $[F(1,12)=2.29, p<.16]$.

\section{Discussion}

The present results clearly indicate that the target on the previous trial played an important role in determining what features captured attention on the current trial. These results are very similar to the results for the uninformative cues in Experiment 1 and also strikingly resemble the typical contingent capture results (Folk et al., 1992). Processing the onset target on trial $n-1$ resulted in capture by onset cues and no capture by color cues on trial $n$. However, when the target on trial $n-1$ was a color target, both onset and color cues captured attention to a similar extent, although there was a small numerical trend for larger capture for the color cues.

Another interesting finding was impaired performance when there was a match between the targets and the cues on any given trial. All the cues captured attention; however, capture was reduced when there was a match between the features of the target and the cue. This is the opposite of what is predicted by both contingent capture and intertrial priming accounts but probably reflects some form of masking arising when a similar feature occurs at the same location close in time. Note, however, that the target on the previous trial leads to the opposite effect.

Although the results of Experiment 2 resemble the contingent capture results, the similarity is not perfect, since capture by the onset cues could not be prevented. This leaves open the possibility that top-down control plays a role in controlling capture by nonmatching features. We did not find evidence for this in Experiment 1, in which the participants received a $100 \%$ informative cue about the upcoming target. However, one possible criticism is that the participants did not fully or consistently use the information that was provided. The only way to make sure that participants are motivated to adopt a specific top-down set on every trial is to create a task that cannot be performed correctly unless the required top-down set is instantiated. Such a task was used in Experiment 3.

\section{EXPERIMENT 3}

The possibility of flexible top-down attentional control was examined in a task that could not be performed with-

Table 1

Reaction Times (RTs, in Milliseconds) and Error Rates (ERs, in Percentages) in Experiment 2

\begin{tabular}{|c|c|c|c|c|c|c|c|c|}
\hline & \multicolumn{4}{|c|}{$\begin{array}{l}\text { Color Target } \\
\text { (Current Trial) }\end{array}$} & \multicolumn{4}{|c|}{$\begin{array}{l}\text { Onset Target } \\
\text { (Current Trial) }\end{array}$} \\
\hline & \multicolumn{2}{|c|}{ Color Cue } & \multicolumn{2}{|c|}{ Onset Cue } & \multicolumn{2}{|c|}{ Color Cue } & \multicolumn{2}{|c|}{ Onset Cue } \\
\hline & RT & $\overline{E R}$ & RT & $\overline{E R}$ & RT & $\overline{E R}$ & RT & $\overline{E R}$ \\
\hline Vali & 458 & 4.2 & 459 & 3.2 & 442 & 3.5 & 442 & 4.8 \\
\hline Invalid & 465 & 7.2 & 483 & 5.6 & 464 & 3.7 & 457 & 4.8 \\
\hline
\end{tabular}


out an appropriate top-down set. The task was very similar to the one used in Experiment 1, except that the neutral instructional cues were omitted and the no-go trials were introduced. Every trial began with an instructional cue (RED or WHITE), which indicated the feature of the target that the participants had to search for. Importantly, on one third of the trials, the participants were presented with the other target display and were required to withhold their response. Therefore, receiving an instructional RED cue meant that the participants had to respond to the color singleton and to withhold their response if the onset singleton was presented. In turn, receiving a WHITE cue meant that they had to respond to the onset singleton and to withhold the response if the color singleton was presented. The participants had to keep the top-down set online until the search display occurred; otherwise, they would make an incorrect response. In order to give the participants sufficient time to generate the appropriate attentional control setting, we also extended the time available to encode and process the instructional cue to $2,500 \mathrm{msec}$. Can the top-down set that was forced to be adopted to optimally perform this task prevent attentional capture by the nonmatching cues?

\section{Method}

Participants. Eleven students ( 8 of them male; mean age $=$ 22 years; range, 17-29 years) participated in the experiment as paid volunteers or in exchange for course credit. One participant was replaced because of an excessive error rate $(>10 \%)$.

Stimuli. The stimuli were exactly the same as those in Experiment 1 , except that the neutral cues were omitted.

Design and Procedure. All the conditions were within subjects and were randomly mixed within blocks. Instructional cues (RED or WHITE) were presented for $2,000 \mathrm{msec}$ and were followed by a fixation screen for another $500 \mathrm{msec}$. Instructional cues informed the participants about which target to search for (go trials). They were also informed that, often, the other type of target display could be presented and were instructed to withhold the response on these trials (no-go trials). In all the conditions, onset or color cues were equally likely to precede the target display, and as before, the cue predicted the target location on a chance level.

The participants received 12 experimental blocks of 48 trials each, preceded by 1 practice block ( 48 trials). In each experimental block, the color- and onset-instructional cues were equally likely to occur. On go trials, which constituted two thirds of all the trials in a block, the participants were instructed to respond quickly and accurately by pressing the " $\mathrm{x}$ " key when the target was " $\mathrm{X}$ " and the " $\mathrm{m}$ " key when the target was " =." It was emphasized that the participants had to withhold their response on the no-go trials. After each block, the participants received feedback about their performance.

\section{Results}

Trials on which the participants responded more quickly than $150 \mathrm{msec}$ or more slowly than $1,200 \mathrm{msec}$ were excluded from further analysis. This led to a loss of $4.5 \%$ of the trials. Mean correct RTs for valid and invalid trials at each combination of cue and target property are presented in Figure 5.

To investigate whether the adopted top-down set influenced attentional capture, mean correct RTs from go trials were submitted to a $2 \times 2 \times 2$ within-subjects ANOVA with target property (color vs. onset), cue property (color vs. onset), and validity (valid vs. invalid) as factors. There was a significant interaction of target and cue properties $[F(1,10)=38.75, p<.005]$, indicating that for both color and onset targets, the responses were faster when the cue property matched the target property, but this difference was more profound for the onset targets. In addition, target property interacted with validity $[F(1,10)=6.61, p<$ $.05]$, indicating that the overall positive effect of cue validity was observed for the color targets but a reversed (negative) validity effect was observed for the onset targets. Importantly, the three-way interaction between target property, cue property, and cue validity was significant $[F(1,10)=20.61, p<.005]$. This interaction suggests that the top-down set influenced which cue property captured attention. However, the pattern of this interaction is very different from the typical contingent capture results (see Figure 5). Further analysis was conducted to determine the effects of the cues that did not match the top-down set.

The influence of cue property on cue validity was further explored in planned comparisons conducted at each level of target property. For the color targets, there was only a main effect of cue validity $[F(1,10)=13.57, p<$ $.005]$. Importantly, the interaction between cue property

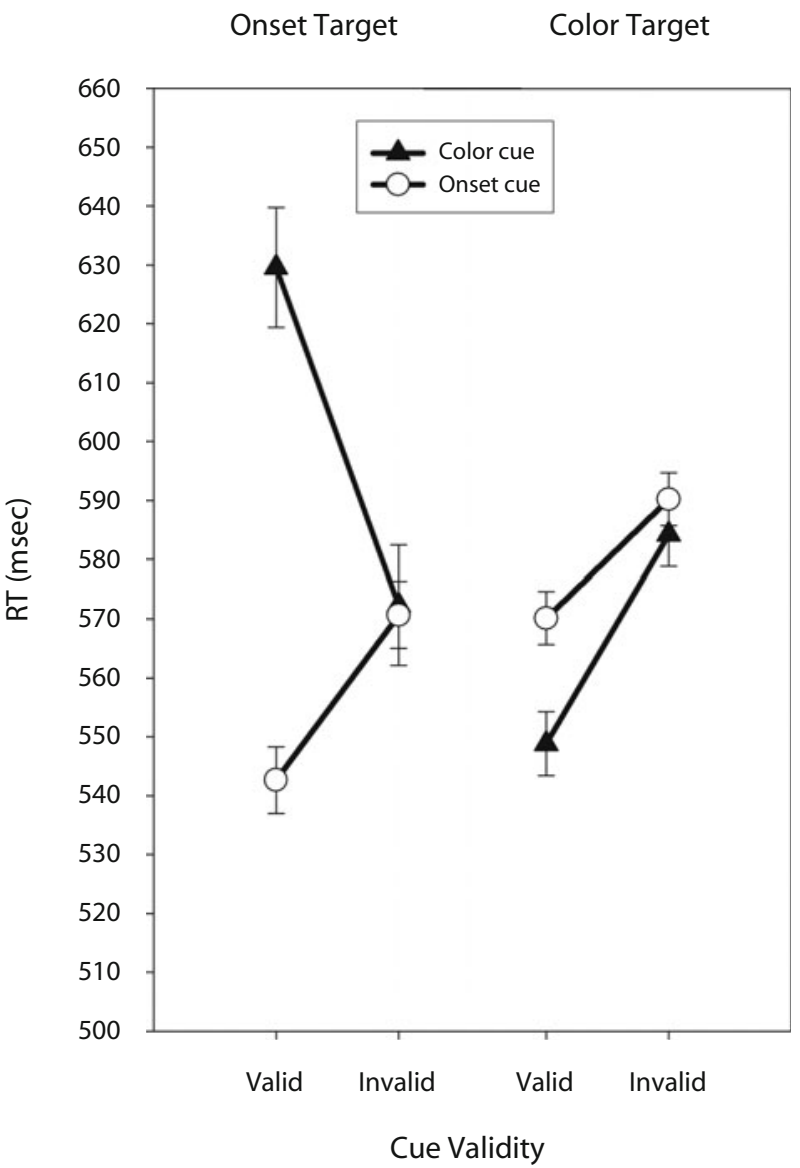

Figure 5. Mean reaction times (RTs) as a function of target property, cue property, and cue validity in Experiment 3. The error bars represent standard errors of the means for withinsubjects designs normalized for the cue validity factor. 
and cue validity was not significant $[F(1,10)=1.40, p=$ .27]. The absence of the interaction suggests that even though the participants adopted a top-down set for color, both color and onset cues resulted in a similar validity effect ( 35 and $20 \mathrm{msec}$, respectively).

For the onset targets, there was only a main effect of cue property $[F(1,10)=12.23, p<.01]$, indicating that trials with onset cues were, overall, responded to more quickly than were trials with color cues. The interaction between cue property and cue validity was significant $[F(1,10)=$ $11.45, p<.01]$, suggesting that onset and color cues captured attention differently. Post hoc tests showed that onset cues captured attention $[28 \mathrm{msec} ; t(10)=2.47, p<$ $.05]$; however, the color cues produced a large significant negative effect of cue validity $[-57 \mathrm{msec} ; t(10)=2.81$, $p<.05]$.

Error rates on the go trials were relatively low (4.4\%), and the ANOVA revealed no significant effects or interactions. False alarm rates on the no-go trials were also low (5.2\%), and no significant effects or interactions were revealed.

\section{Discussion}

The present results demonstrate that foreknowledge about the target property does modulate the processing of the irrelevant cue property. In this experiment, we extended the presentation time of the instructional cue so that the participants had sufficient time to adopt the top-down set. The design of the task also enabled us to check whether the participants would adopt the required top-down set; otherwise, they could not perform the task correctly.

However, the mechanism of the top-down modulation observed in the present experiment is drastically different from the mechanism postulated by the contingent capture hypothesis (Folk et al., 1992). The results actually resemble those of Experiment 1 in illustrating that attentional capture by the cues that do not match the top-down set cannot be prevented. As in Experiment 1, when the participants were set for color targets, both color and onset cues captured attention. When the participants were set for onset targets, only onset cues captured attention. Interestingly, adopting a top-down set for onset targets also led to suppression of color cues in this condition. Such suppression suggests that attention was captured by the nonmatching cue but was quickly disengaged from its location, because the participants realized that the color cue was not the feature they were looking for. As a consequence of this disengagement, the participants were slower to respond when the target happened to be presented at that location. Perhaps the role of top-down control is precisely this: to disengage attention from the locations of features that do not match the top-down set (see Theeuwes, Atchley, \& Kramer, 2000).

We believe that the design of Experiment 3 encouraged the participants to adopt a top-down attentional set on every trial and allowed us to check whether that was the case. However, finding attentional capture by irrelevant cues in our paradigm is always subject to the criticism that the top-down set was not fully established, maintained, or both. Indeed, so far, we have assumed that providing participants with foreknowledge about their search and response target is sufficient for establishing a top-down attentional set. Note that the same logic is also used in the classic version of the contingent capture paradigm, which does not incorporate any procedures to check whether participants actually establish or actively maintain the required top-down set on every trial. In fact, most paradigms are designed in a way that it would be either advantageous for participants to follow the instructions or impossible for them to accomplish the task correctly if the instructions are not followed. Nevertheless, it can only be assumed that on any particular trial, participants actually make a conscious decision to reestablish a certain top-down set according to the given instructions. The design of Experiment 3 certainly demanded such reestablishment of the top-down set to a greater extent than did Experiment 1 or the classic version of the contingent capture paradigm. To further encourage participants to establish an attentional set on every trial, in Experiment 4, we partially gave them their "free will" back and asked them to choose themselves which target they wanted to search for.

Although, in Experiment 3, we included a checking procedure (go/no-go task) to ensure that the participants indeed remembered what the top-down set was, we could not check whether the appropriate attentional set was continuously maintained throughout each trial. It would certainly have been advantageous for the participants to actively maintain the attentional set, but it is still plausible that its strength fluctuated during the trial. Lapses in the attentional set, if they occurred, would have resulted in attentional capture by the irrelevant cues. This was indeed the case for the top-down set for color targets, but suppression of the irrelevant color cues was found for the onset targets. This discrepancy suggests that, perhaps due to such fluctuations, the strength of top-down control in Experiment 3 was not sufficient to suppress processing of such salient features as abrupt onsets. To counteract fluctuations in top-down control in Experiment 4, we asked participants to vocally rehearse the chosen attentional set during the trial.

\section{EXPERIMENT 4}

Experiment 4 was similar to Experiment 3, except that we gave the participants a choice of which attentional set they wanted to establish and asked them to repeat out loud the chosen target feature until the search display appeared. The participants were also free to choose when to start the trial and to decide for themselves how much time they needed to establish the top-down set. Asking participants to rehearse attentional set vocally should minimize reliance on working memory and prevent potential lapses of attentional set associated with lapses in working memory maintenance (Baddeley, 1986; Peterson \& Peterson, 1959). Therefore, in the present experiment, on every trial the participants were given every opportunity to establish and maintain their topdown control for a specific feature and, thereby, optimize their search for the target. Would such extensive top-down control be enough to avoid distraction by the irrelevant 
cues, as is suggested by the contingent capture hypothesis? Or would top-down control operate by suppression of all irrelevant cues, as was indicated in Experiment 3?

\section{Method}

Participants. Thirteen students ( 8 of them male; mean age $=$ 22 years; range, $19-25$ years) participated in the experiment as paid volunteers or in exchange for course credit.

Stimuli, Design, and Procedure. The experiment was very similar to Experiment 3, except that before the start of each trial, the participants were presented with a display with a two-choice menu in the middle and were asked to choose whether they wanted to search for an onset or a color target. They had to press " 1 " if they wanted to search for the red target and "2" if they wanted to search for the white target. As soon as the participants pressed the corresponding number with their left hand, the font of the chosen option increased in size, providing the participants with immediate feedback about which option was selected. In the top right corner of the choice display, there were two bars, labeled red and white, depicting the cumulative number of trials on which each target had been chosen within a block. The participants were asked to choose an approximately equal number of onset and color targets in each block and to keep the corresponding bars as equal as they could. If they chose the same target three times in a row, they received a warning message; however, they were not prevented from choosing the same option again. After a choice was made, the choice display with the selected option was presented for $200 \mathrm{msec}$, and after a blank screen of $500 \mathrm{msec}$, the same fixation screen as that in Experiment 3 appeared for a randomly chosen duration of 1,000 $1,400 \mathrm{msec}$. The rest of the stimuli were identical to those in Experiment 3. The participants were asked to repeat the chosen top-down set ("red" or "white") out loud at their own pace until making a response. The vocal rehearsal was recorded using a digital recorder.

On $50 \%$ of the trials, the participants saw the target that they had chosen to respond to (go trials). They were also informed that, often, the other target could be presented and were instructed to withhold the response on these trials (no-go trials). In all the conditions, onset or color cues were equally likely to precede the target display, and as before, the cue predicted the target location on a chance level.

The participants received eight experimental blocks of 96 trials each, preceded by one practice block ( 32 trials). On go trials, the participants were instructed to respond quickly and accurately to the chosen target by pressing the " 0 " key when the target was " $\mathrm{X}$ " and the "." key when the target was "=." It was emphasized that the participants had to withhold their response on the no-go trials, when the target that they had not chosen appeared. After each block, the participants received feedback about their performance.

\section{Results}

Trials on which the participants responded more quickly than $150 \mathrm{msec}$ or more slowly than $1,400 \mathrm{msec}$ were excluded from further analysis. This led to a loss of 5\% of the trials. On average, the participants were equally likely to choose the color and onset targets $(t<0.1)$.

Mean correct RTs for valid and invalid trials at each combination of cue and target property are presented in Figure 6. To investigate whether the adopted top-down set influenced attentional capture, mean correct RTs from go trials were submitted to a $2 \times 2 \times 2$ within-subjects ANOVA with target property (color vs. onset), cue property (color vs. onset), and cue validity (valid vs. invalid) as factors. There was a significant main effect of cue property $[F(1,12)=9.22, p<.05]$, indicating that the participants tended to respond more quickly when the onset cue was present. In addition, there was an interaction of target and cue properties $[F(1,12)=33.10, p<.001]$, indicating that for both color and onset targets, the responses were faster when the cue property matched the target property, but this difference was more profound for the onset targets. Importantly, the three-way interaction between target property, cue property, and cue validity was significant $[F(1,12)=28.64, p<.001]$. This interaction suggests that the top-down set influenced how each cue property was spatially processed. However, the pattern of this interaction is very different from the typical contingent capture results and suggests that for both types of targets, only the cues matching the target property captured attention, whereas the nonmatching cues were suppressed (see Figure 6). Further analysis was conducted to determine the effects of the cues that did not match the top-down set.

The influence of cue property on cue validity was further explored in planned comparisons conducted at each level of target property. For the color targets, there was a main effect of cue property $[F(1,12)=11.09, p<.01]$, indicating that trials with color cues were, overall, responded to more quickly than were trials with onset cues. Importantly, the interaction between cue property and cue validity was significant $[F(1,12)=27.71, p<.001]$.

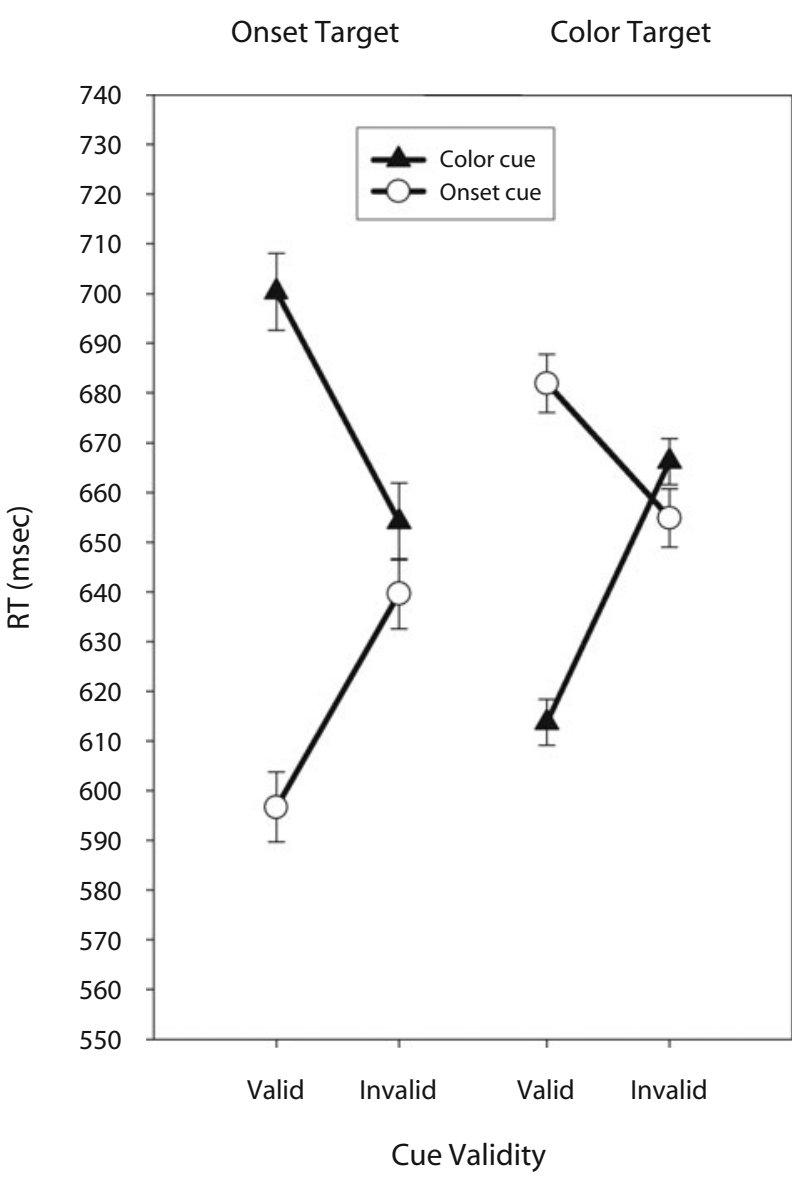

Figure 6. Mean reaction times (RTs) as a function of target property on the previous trial, cue property, and cue validity in Experiment 4. The error bars represent standard errors of the means for within-subjects designs normalized for the cue validity factor. 
Post hoc tests showed that color cues captured attention [52 msec; $t(12)=5.68, p<.001]$; however, the onset cues produced a significant negative effect of cue validity $[-27 \mathrm{msec} ; t(12)=2.32, p<.05]$.

For the onset targets, there was a main effect of cue property $[F(1,12)=37.12, p<.001]$, indicating that trials with onset cues were, overall, responded to more quickly than were trials with color cues. The interaction between cue property and cue validity was significant $[F(1,12)=$ $17.36, p<.01]$, suggesting that onset and color cues captured attention differently. Post hoc tests showed that onset cues captured attention [43 msec; $t(12)=3.05, p<.05]$; however, the color cues produced a significant negative effect of cue validity [ $-46 \mathrm{msec} ; t(12)=2.98, p<.05] .{ }^{1}$

Error rates on the go trials were relatively low (4\%), and the ANOVA revealed no significant effects or interactions. False alarm rates on the no-go trials were also low $(2.5 \%)$, and no significant effects or interactions were revealed.

\section{Discussion}

The results of the present experiment clearly demonstrate that top-down control was adopted on a trial-bytrial basis and had a profound influence on search performance. Consistent with the contingent capture hypothesis, both onset and color cues produced a positive cuing effect only when they matched the top-down set. However, the cues that did not match the top-down set were not simply filtered out but were suppressed, which is inconsistent with the contingent capture hypothesis. Targets occurring at the location of nonmatching cues were detected more slowly, as compared with when they occurred at the uncued locations (negative cuing effect). For the color cues that did not match the top-down set, such suppression has already been reported in Experiment 3, but here we extended this result to the onset cues that did not match the attentional set for color. This was achieved by strengthening the top-down set for a specific feature by asking participants to choose the target feature themselves and by maintaining this set using overt rehearsing. Suppression of the nonmatching cue location suggests that top-down control could not prevent attentional capture by the cue but operated by rapid disengagement of attention from the cued location, which was followed by suppression of processing at that location (Theeuwes et al., 2000).

The role of top-down control in attentional capture becomes clear when the results of Experiment 4 are directly compared with the results of Experiment 1 . In Experiment 1 , the participants were fully informed about the feature of the upcoming target but did not have to use this information to perform the task. Such a weak top-down set resulted in a positive cuing effect for the irrelevant cues (somewhat smaller than that for the relevant cues), indicating that attention was allocated and still maintained at the nonmatching cue location at the time the target was presented. Adopting a very strong top-down set for a specific property that was required in Experiment 4 resulted in attention's being quickly withdrawn from the location of the cue that did not match that property. This compari- son suggests that when the top-down set is weak, attention tends to linger at the irrelevant cue location, but strengthening the top-down set speeds up the disengagement of attention from that location and leads to spatially selective suppression at that location.

\section{GENERAL DISCUSSION}

The present study challenges the assumptions of one of the most influential hypotheses in attention research of the last 15 years. According to the contingent capture hypothesis, task goals determine what features of the environment are of immediate importance. The hypothesis states that "under conditions of spatial uncertainty, the exogenous allocation system can be 'configured' or 'set' to respond selectively to a property that signals the (unpredictable) location of stimuli that are relevant to optimal task performance. Thus, any particular system configuration, or 'attentional control setting,' is assumed to be a function of current behavioral goals" (Folk et al., 1992, p. 1041). It is assumed that features that do not match the current behavioral goals are simply filtered out (Folk \& Remington, 1998).

The challenge of the present study to the contingent capture hypothesis rests on two main conclusions. First, it appears that top-down control does not operate by filtering out salient objects that are irrelevant to the goal of the observer. Instead, it operates by rapid disengagement from the salient stimuli that do not match the top-down set, which is followed by suppression of processing at that location (Experiments 3 and 4). Therefore, we suggest that top-down control is not able to prevent initial attentional capture by nonmatching salient stimuli but, instead, allows a fast disengagement from an attended location. Second, bottom-up intertrial priming can produce results that are very similar to the contingent capture results, without the need to postulate a role for top-down influence (Experiments 1 and 2).

\section{The Role of Top-Down Control in Attentional Capture}

In the present study, we gave observers full opportunity to set themselves optimally for task performance on every trial. This was accomplished either by providing them with a $100 \%$ valid instructional cue about the target property (Experiment 1) or with an instructional cue about the target property that was critical for performing the task (Experiment 3 ) or even by letting them choose for themselves which target property to search for (Experiment 4). Nevertheless, in all cases, spatially selective processing of the irrelevant cue took place. In Experiment 1, both irrelevant onset and color cues captured attention, and in Experiment 3, only irrelevant onset cues captured attention, whereas the color cues were suppressed. In Experiment 4 , the locations of both the irrelevant onset and the color cues were suppressed when they did not match the top-down set. This is inconsistent with the contingent capture hypothesis (Folk \& Remington, 1998; Folk et al., 1992; Folk et al., 1994), which postulates that only infor- 
mation that matches the attentional set captures attention and the salient nonmatching information can be nonspatially filtered out.

The difference between previous studies showing contingent capture and the present study is that in the previous studies, attentional set was fixed over a block of trials, allowing observers to consistently search for the same target, whereas in the present study, attentional set for the upcoming trial needed to be instantiated on each trial. We specifically used a more abstract word cue, instead of a symbolic cue showing the actual color or onset, to make sure that the cue could not generate priming of any of the features of the upcoming target (see, e.g., Theeuwes \& Van der Burg, 2007, who showed that a symbolic cue caused bottom-up priming). If, as is claimed by the contingent capture hypothesis, volitional top-down control determines attentional capture, there is no reason to assume that instantiating these settings at the beginning of each trial would render different results. Indeed, at the heart of the contingent capture hypothesis is the assumption that involuntary attentional orientation depends on voluntary control settings determined by the task constraints. In our study, we presented these task constraints at the beginning of each and every trial. If people are able to voluntarily set themselves for a particular task, this should have occurred in the present study. Indeed, if anything, the contingent effects should have only been stronger, because the top-down set was reinforced on every trial. One could argue that in Experiment 1, the observers did not follow the task instructions and simply chose not to be prepared for the upcoming trial. Even though this is possible, it should be realized that in Posner-like location cuing experiments, observers typically follow instructions without any problems and show a great deal of top-down control over visual selection. Our Experiments 3 and 4 make an even stronger case for observers' using the information to set themselves for the upcoming trial, because this information was necessary for them to know what target they had to respond to.

Obviously, our data are inconsistent with contingent capture, showing that such an attentional control setting did not affect involuntary attentional orienting. We show that it is impossible to filter out irrelevant salient stimuli by providing the top-down set on a trial-by-trial basis. Interestingly, in a recent study, Lien, Johnston, and Ruthruff (in press) showed that top-down set for a specific target color adopted on a trial-by-trial basis could modulate and, in some cases, prevent capture by irrelevant color singletons and abrupt onsets. Although further research is needed to explain the discrepancies between these results, it is worth noting that in the Lien et al. (in press) study, the crucial validity effect when the color of the cue matched the color of the target was driven primarily by the invalid condition. In other words, it seems that observers had trouble disengaging attention from the invalid location when the color at that location matched the color they were looking for. Because the observers had no trouble disengaging attention when the color of the cue did not match the color of the target, no such validity effect was found in the nonmatching condition. Even though the general pattern of results in Lien et al. (in press) is similar to the classic findings of Folk et al. (1992), there are particularities in the data, including very long overall RTs, that suggest that processes other than contingent capture may have been responsible for these effects.

Note that the argument that observers choose not to adopt a top-down set on a trial-by-trial basis is simply incorrect. Our data provide clear evidence that a top-down set adopted on a trial-by-trial basis influenced the processing of the irrelevant salient cue. When the observers were set for onset targets, irrelevant color cues either captured attention less than did relevant onset cues (Experiment 1) or produced spatially selective suppression of target processing (Experiment 3). When the participants chose the target feature themselves and overtly rehearsed the topdown set (Experiment 4), spatially selective suppression was found for all cues that did not match the top-down set, whereas all the cues that did match the top-down set captured attention. A similar spatially selective suppression effect was also reported but not brought into discussion in a recent study by Folk and Remington (2008). In their Experiment 2, during the whole experiment, participants were asked to search for and respond only to the targets of a specific color (i.e., red) and to withhold a response to targets of a different color (i.e., green). They found that whereas the color cues that matched the top-down set for a specific color captured attention, the nonmatching color cues revealed a small but significant negative cue validity effect $(-9 \mathrm{msec})$. In our Experiment 2, we found a much larger suppression effect for irrelevant color cues $(57 \mathrm{msec})$. This large difference in the magnitude of the effect could be due to the fact that, in our experiment, the top-down set had to be instantiated on every trial, whereas in a study by Folk and Remington (2008), the top-down set had to be maintained for the entire experiment. It is feasible that in the latter case, there were larger fluctuations in top-down control, as compared with the former case, when the required top-down set was reiterated on every trial.

Clearly, the suppression of irrelevant cues found in Experiments 3 and 4, as well as in Folk and Remington (2008), represents a form of top-down control. Note, however, that it is a very different form of control than the one suggested by the contingent capture hypothesis. According to the contingent capture hypothesis, the cues that do not match the top-down set simply do not cause involuntary attentional shifts (Folk et al., 1992). Such cues are filtered out, which sometimes results in a nonspatial filtering cost (Folk \& Remington, 1998). However, in our Experiments 3 and 4, we found spatially selective suppression at the location that contained a feature that did not match the top-down set. We assume that such suppression can take place only after attention has been at that location (for a different view, see Lamy \& Egeth, 2003). Although there was no direct evidence for disengagement of attention in Experiments 3 and 4, such an assumption is valid when the experiments in the present study are considered together. Experiment 1, which did not implement a top-down set as rigorous as those in Experiments 3 and 4, showed that at the moment the target display is present, attention still resides at the nonmatching location. We assume that due 
to the more rigorous top-down set in Experiments 3 and 4, this initial capture resulted in suppression at the location of the nonmatching feature. Furthermore, in a recent study, we showed that it is impossible to detect even the simplest salient feature without focal attention (Theeuwes, Van der Burg, \& Belopolsky, 2008). In other words, it seems that in the present study, in order to decide that a location contained a feature that did not match the top-down set, spatial attention was needed at that location.

A few studies have reported a similar type of suppression at the location of the irrelevant singletons; however, these findings are mostly restricted to suppression of color singletons. For example, using a probe dot technique, Kim and Cave (1999) showed that early in time (60 msec after display onset), attention is directed to the location of a salient distractor, but already by $150 \mathrm{msec}$, attention is switched to the target location. In fact, this is consistent with the proposal of Theeuwes et al. (2000), who suggested that during early visual processing, attention is driven entirely by salience of the cues but that top-down control can be used to quickly disengage attention from the cue that does not match the top-down set. The authors manipulated the SOA between the irrelevant color singleton and the unique shape target display and showed that the color singleton produced interference only when it preceded the target display by no more than $150 \mathrm{msec}$. Furthermore, when such a color singleton contained a letter compatible or incompatible with response to the target, a negative compatibility effect was found at a 200-msec SOA, whereas a positive compatibility effect was observed at earlier SOAs. It is likely that such a suppression of singleton location occurred after attention had been disengaged from the location of the irrelevant singleton.

A very similar pattern of suppression of the color singletons, using Folk's precuing paradigm, has recently been reported by Lamy and colleagues (Lamy \& Egeth, 2003; Lamy, Leber, \& Egeth, 2004). They consistently showed that location of the irrelevant color cue was inhibited even when it preceded the target display by only $60 \mathrm{msec}$. Interestingly, such suppression was present only for color cues, but not for abrupt onsets, which always captured attention (Lamy \& Egeth, 2003). This is similar to the results of the present study, in which, in Experiment 3, suppression was observed only for the color cues and, under similar conditions, it was impossible to suppress the onset cues. However, additional manipulations to boost top-down set in Experiment 4 also led to spatially selective suppression of onset cues. Obviously, onset cues are harder to ignore than color cues, a finding consistent with a large literature showing that abrupt onsets have a special status in capturing attention (e.g., Jonides \& Yantis, 1988; Schreij et al., 2008; Theeuwes, 1990, 1994, 1995; Theeuwes et al., 1998; Yantis \& Jonides, 1984).

Our results provide important insights into the role of top-down control in attentional capture. In line with the disengagement hypothesis (Theeuwes et al., 2000), we propose that top-down control operates through disengagement of attention from the location of the salient irrelevant stimuli. If top-down control is not fully established on every trial, attention might linger at the location of the irrelevant cue on some trials, resulting either in a small positive cuing effect or in a nonsignificant cuing effect. However, if top-down control is established consistently on every trial, attention can be rapidly disengaged from the cued location before presentation of the target, resulting in suppression of processing at that location. The speed and consistency of disengagement of attention from the location of the irrelevant cue depends on the strength and fluctuations of the top-down set. In fact, such a switch from positive to negative cuing effects can be illustrated if our Experiments 1, 3, and 4 are viewed as a continuum for the involvement of the top-down control. Top-down control was probably the weakest in Experiment 1, in which the participants did not have to use the instruction cue; it was probably already quite strong in Experiment 3, in which only irrelevant onsets produced positive cuing effects; and it was the strongest in Experiment 4, in which all the irrelevant cues were suppressed. Such suppression of irrelevant cue location might be similar to inhibition of return, which has been shown with both abrupt onsets and color singletons and with short and long SOAs, depending on the task demands (Danziger \& Kingstone, 1999; Klein, 2000; Theeuwes \& Godijn, 2002).

It is important to note that some recent studies have called into question the disengagement hypothesis by showing that the N2pc component of the event-related potential (ERP), which is often thought to represent shifts of attention, was elicited only by the cues that matched the topdown set, but not by the nonmatching cues (Eimer \& Kiss, 2008; Leblanc, Prime, \& Jolicœur, 2008; Lien, Ruthruff, Goodin, \& Remington, 2008). Although it is an important finding, the absence of $\mathrm{N} 2 \mathrm{pc}$ does not necessarily indicate the absence of shifting attention to nonmatching cue location. According to a recent study by Kiss, Van Velzen, and Eimer (2008), N2pc mainly represents target-related processing and does not represent the actual shifting of attention. Therefore, the absence of N2pc is fully consistent with the disengagement hypothesis, suggesting that an attentional shift to the nonmatching cue location takes place but, because the distractor feature does not match the target feature, the processing at that location is minimal. In other words, consistent with Kiss et al., the presence or absence of an N2pc does not say anything about attentional capture - that is, the shifting of attention to the distractor location. If anything, it indicates whether processing took place at the location of the distractor. If this processing is minimal, the ERP signal will not reveal the N2pc.

\section{The Role of Intertrial Priming in Attentional Capture}

Our analysis of intertrial effects in Experiments 1 and 2 provides additional insights regarding the origin of contingent capture effects. The results indicate that when the target on the current trial was unknown, the target on the previous trial had a large influence on which cue property would capture attention on the current trial. If the target on the previous trial was an abrupt onset, only onset cues captured attention on the following trial, whereas the color cues were ignored. A similar trend was also found for the color targets on the previous trial, with color cues on the 
current trial capturing attention and onset cues capturing attention somewhat less (Experiment 2). As was mentioned earlier, abrupt onsets are typically more salient than color singletons and are harder to ignore (e.g., Jonides \& Yantis, 1988; Schreij et al., 2008; Theeuwes, 1990, 1994, 1995; Theeuwes et al., 1998; Yantis \& Jonides, 1984), and the contingent capture effect with irrelevant onset cues is not always replicated (Lamy \& Egeth, 2003). In fact, abrupt onsets were found to capture attention even when the participants were forced to adopt a top-down set for color (see our Experiment 3).

These results demonstrate that contingent capture can be driven by the target on the previous trial, instead of a top-down set for a particular target property. This is fully consistent with the way most contingent capture experiments have been conducted, since in these experiments, the target remained consistently the same over a whole block of trials. Even though it is assumed that contingent capture effects are caused by a top-down attentional set, the present analysis is consistent with the idea that the effects reported in the original Folk et al. (1992) contingent capture experiments were highly influenced by intertrial priming (for a similar observation, see Becker, Ansorge, $\&$ Horstmann, 2009).

Importantly, previous studies have suggested that such intertrial effects are largely due to bottom-up priming (e.g., Kristjánsson, Wang, \& Nakayama, 2002; Pinto, Olivers, \& Theeuwes, 2005; Theeuwes et al., 2006; Theeuwes $\&$ Van der Burg, 2007, 2008). For example, Maljkovic and Nakayama (1994) investigated a mechanism referred to as priming of pop-out. In their task, participants searched for a feature singleton that was defined in two different ways: a red target among green distractors or a green target among red distractors. They showed that even when repetition of the feature value was at chance level (i.e., repetition was no more likely than alternation), repeating a target (but not the response) improved performance (for the neural correlates of this effect, see Kristjánsson, Vuilleumier, Schwartz, Macaluso, \& Driver, 2007). Maljkovic and Nakayama concluded that this effect was due to automatic visual priming, which is basically not affected by the top-down set. Kristjánsson et al. (2002) reported intertrial priming effects in conjunction visual search. Kristjánsson et al. (2002) concluded that "the role of priming in visual search is underestimated in current theories of visual search and that differences in search times often attributed to top-down guidance may instead reflect the benefits of priming" (p. 37).

The importance of intertrial effects in contingent capture was also acknowledged in the study by Folk and Remington (2008). In Experiment 1 of their study, participants were set to adopt a general set for color (i.e., to look for either green or red color singletons). The search display was preceded by uninformative red and green color cues. It was found that both red and green color cues captured attention. However, the results also showed that attentional capture was modulated by the color of the target on the previous trial. Basically, the capture was greater when the color of the cue on the current trial matched the color of the target on the previous trial. However, the target on the previous trial did not fully determine which feature would capture attention on the current trial. For example, when the target on the previous trial was a green singleton, capture by red cues was reduced but not abolished. In addition, in Experiment 2 in Folk and Remington (2008), participants were asked to search for and respond only to the targets of a specific color (i.e., red) and to withhold responses to targets of a different color (i.e., green). Such a narrowly defined top-down set led to the finding that only relevant matching cues produced a positive cuing effect. Intertrial priming effects also disappeared, which the authors explained by suggesting that bottom-up priming exists only when the primed feature value is left unspecified in the control settings. On the basis of these two experiments, the authors concluded that bottom-up priming can affect top-down control settings but cannot fully explain contingent capture.

One important difference from our results is that in Experiment 1 in Folk and Remington (2008), cues that did not match the color of the previous target still captured attention. However, in our study, capture by color cues was completely eliminated when the previous target was an onset. This result, similar to the typical contingent capture result, however, is achieved on a single trial and is fully driven by intertrial priming. This discrepancy could be due to the fact that we asked participants to search for a singleton across dimensions (color or onset), which might be different from the search for a feature singleton within the color dimension that was required by Folk and Remington (2008). Note that it has previously been demonstrated that irrelevant cues within a feature dimension do capture attention even when the attentional set is fixed (Folk et al., 1992, Experiment 4).

Intertrial priming is thought to guide visual selection by adjusting the weights for the target and distractor features, particularly in situations in which there is competition for attentional selection (Meeter \& Olivers, 2006; Müller, Heller, \& Ziegler, 1995; Olivers \& Humphreys, 2003; Olivers \& Meeter, 2006). On the basis of the previous experience, features associated with targets receive more weight, whereas features associated with distractors receive less weight. These intertrial adjustments seem to occur in automatic fashion, and recent studies have shown that what is transferred is the target selection and distractor rejection process that occurred on the previous trial (Becker, 2007; Hillstrom, 2000; Wolfe, Butcher, Lee, \& Hyle, 2003). For example, Geyer, Müller, and Krummenacher (2006) showed that in a multiple color-orientation conjunction search, observers adopted a color-subset-based search strategy, looking for the odd-one-out orientation in a given color subset. Importantly, the repetition of distractor features only within the selected color subset, and not in the ignored color subset, sped up the RTs on the next trial. Such modulation of intertrial priming by task set suggests that it lies somewhere in between the purely top-down and purely bottom-up control, most likely reflecting an automatic transfer of a target selection process on the previous trial. Critically, this is different from the type of control assumed by the contingent capture hypothesis, which postulates that a top-down set established without immediate experience with the target 
selection process can prevent attentional capture by irrelevant features (Lien et al., in press).

Previous studies have shown that intertrial priming can modulate attentional capture by salient irrelevant distractors. For example, Pinto et al. (2005) showed that priming the target feature reduces interference from the salient distractor (see also Theeuwes \& Van der Burg, 2008). In addition, it has been shown that previous exposure to a salient distractor reduces or even eliminates its interference on the following trial (Geyer, Müller, \& Krummenacher, 2008; Müller, Geyer, Zehetleitner, \& Krummenacher, 2009). Therefore, intertrial priming can, to a large extent, explain the contingent capture in the typical precuing paradigm, in which the target feature is constant in a block of trials and the distracting cues are present on every trial (Folk et al., 1992). The residual capture by irrelevant onsets found in the present study can be explained by a recent suggestion that the weight of a distractor feature cannot be modulated to nothing: Finding residual capture or absence of capture depends on how salient the distractor feature is (Müller et al., 2009).

Another possibility is that the lack of cuing effect of the onset cue in the typical contingent capture paradigm (but see Lamy \& Egeth, 2003) is, in fact, due to some form of top-down control, operating together with intertrial priming. Such top-down control for a specific target feature was not possible in our Experiment 2, since the participants were set for both color and onset targets. Recent studies have suggested that top-down effects do play a role in the modulation of attentional capture (Geyer et al., 2008; Müller et al., 2009). However, the results from Experiments 3 and 4 suggested that the mechanism of top-down control is different from intertrial priming. Whereas intertrial priming operates through adjustment of weights, top-down control operates postselectively, through disengagement of attention. Therefore, we propose that top-down control might be additive to the intertrial priming effects.

Such a conclusion is consistent with the results recently reported by Theeuwes and Van der Burg (2008). They showed that a verbal top-down cue presented on a trialby-trial basis reduced search time in the distractor-present condition by $41 \mathrm{msec}$, as compared with a neutral cue. Presenting participants with a symbolic cue identical to the target reduced the search time by $70 \mathrm{msec}$. Since both top-down and intertrial priming were involved in the latter condition, the authors suggested that top-down and intertrial effects were additive and intertrial priming resulted in an additional 29-msec reduction in RT.

In summary, according to the contingent capture hypothesis, attentional set determines what features of the environment will capture attention. We have shown that even when participants adopted a top-down attentional set on a trial-by-trial basis, they could not prevent attentional capture by the irrelevant cue. Forcing participants to adopt a top-down set on every trial sped disengagement of attention and led to suppression of the location of the irrelevant cue. We assume that this spatially selective suppression occurs after attention has been captured by the irrelevant cue. We propose that the primary role of the top-down set is to control the disengagement of attention from the fea- tures that do not match it. The speed of disengagement is determined by the strength of the top-down set: If the topdown set is strong, attention will be quickly disengaged from the salient item that does not match it; if the top-down set is weak, attention will linger at that location. In addition, our results suggest that the classic findings showing contingent capture were strongly influenced by bottom-up priming by the target processed on the previous trial. Our results suggest that the concept of attentional set as currently proposed by the contingent capture hypothesis on the basis of the precuing paradigm is not purely top-down. In fact, when the search target is fixed, voluntary top-down control does not have to be present in a continuous fashion on each trial during the search task; that would make our behavior very slow and would require a lot of resources. We speculate that once the target parameters defined by instruction have led to a correct response, attentional selection can carry on on the basis of the bottom-up intertrial priming. This means that voluntary control over selection is not necessary and attentional set is established after the first few experiences with the target. The function of topdown control is reduced to monitoring-verification and checking of whether the selected information matches the goal, as well as disengagement-but top-down control itself does not control the selection process.

\section{AUTHOR NOTE}

This research was funded by Grant HSFP-RGP39/2005 from the Human Frontier Science Program to J.T. We thank Árni Kristjánsson, Richard Abrams, Chip Folk, Thomas Geyer, Hermann Müller, and Steve Luck for their very helpful comments on this article. We also thank Bradley Gibson for his comments and suggestions as the action editor. Correspondence concerning this article should be addressed to A. V. Belopolsky, Department of Cognitive Psychology, Vrije Universiteit, van der Boechorststraat 1, 1081 BT Amsterdam, The Netherlands (e-mail: a.belopolsky@psy.vu.nl).

\section{REFERENCES}

Baddeley, A. D. (1986). Working memory. Cambridge, MA: MIT Press.

BECKER, S. I. (2007). Irrelevant singletons in pop-out search: Attentional capture or filtering costs? Journal of Experimental Psychology: Human Perception \& Performance, 33, 764-787.

Becker, S. I., Ansorge, U., \& Horstmann, G. (2009). Can intertrial priming account for the similarity effect in visual search? Vision Research, 49, 1738-1756.

Burnham, B. R. (2007). Displaywide visual features associated with a search display's appearance can mediate attentional capture. Psychonomic Bulletin \& Review, 14, 392-422.

Danziger, S., \& Kingstone, A. (1999). Unmasking the inhibition of return phenomenon. Perception \& Psychophysics, 61, 1024-1037.

Eimer, M., \& Kiss, M. (2008). Involuntary attentional capture is determined by task set: Evidence from event-related brain potentials. Journal of Cognitive Neuroscience, 20, 1423-1433.

Folk, C. L., \& Remington, R. W. (1998). Selectivity in distraction by irrelevant featural singletons: Evidence for two forms of attentional capture. Journal of Experimental Psychology: Human Perception \& Performance, 24, 847-858.

Folk, C. L., \& Remington, R. W. (2008). Bottom-up priming of topdown attentional control settings. Visual Cognition, 16, 215-231.

Folk, C. L., Remington, R. W., \& Johnston, J. C. (1992). Involuntary covert orienting is contingent on attentional control settings. Journal of Experimental Psychology: Human Perception \& Performance, 18, 1030-1044.

Folk, C. L., Remington, R. W., \& Wright, J. H. (1994). The structure of attentional control: Contingent attentional capture by apparent mo- 
tion, abrupt onset, and color. Journal of Experimental Psychology: Human Perception \& Performance, 20, 317-329.

Geyer, T., Müller, H. J., \& Krummenacher, J. (2006). Cross-trial priming in visual search for singleton conjunction targets: Role of repeated target and distractor features. Perception \& Psychophysics, 68, 736-749.

Geyer, T., Müller, H. J., \& Krummenacher, J. (2008). Expectancies modulate attentional capture by salient color singletons. Vision Research, 48, 1315-1326.

Hickey, C., McDonald, J. J., \& Theeuwes, J. (2006). Electrophysiological evidence of the capture of visual attention. Journal of Cognitive Neuroscience, 18, 604-613.

Hillstrom, A. P. (2000). Repetition effects in visual search. Perception \& Psychophysics, 62, 800-817.

JONIDES, J., \& YANTIS, S. (1988). Uniqueness of abrupt visual onset in capturing attention. Perception \& Psychophysics, 43, 346-354.

Kim, M.-S., \& CAVE, K. R. (1999). Top-down and bottom-up attentional control: On the nature of interference from a salient distractor. Perception \& Psychophysics, 61, 1009-1023.

Kiss, M., Van Velzen, J., \& Eimer, M. (2008). The N2pc component and its links to attention shifts and spatially selective visual processing. Psychophysiology, 45, 240-249.

KLEIN, R. M. (2000). Inhibition of return. Trends in Cognitive Sciences, 4, 138-147.

Kristjánsson, Á., Vuilleumier, P., Schwartz, S., Macaluso, E., \& Driver, J. (2007). Neural basis for priming of pop-out during visual search revealed with fMRI. Cerebral Cortex, 17, 1612-1624.

Kristuánsson, Á., Wang, D., \& NaKayama, K. (2002). The role of priming in conjunctive visual search. Cognition, 85, 37-52.

Lamy, D., \& Egeth, H. E. (2003). Attentional capture in singletondetection and feature-search modes. Journal of Experimental Psychology: Human Perception \& Performance, 29, 1003-1020.

LAMY, D., Leber, A., \& EGETH, H. E. (2004). Effects of task relevance and stimulus-driven salience in feature-search mode. Journal of Experimental Psychology: Human Perception \& Performance, 30, 1019-1031.

Leblanc, E., Prime, D. J., \& Joliceur, P. (2008). Tracking the location of visuospatial attention in a contingent capture paradigm. Journal of Cognitive Neuroscience, 20, 657-671.

Lien, M. C., Johnston, J. V., \& RuthrufF, E. (in press). Attentional capture with rapidly changing attentional control settings. Journal of Experimental Psychology: Human Perception \& Performance.

Lien, M. C., Ruthruff, E., Goodin, Z., \& Remington, R. W. (2008). Contingent attentional capture by top-down control settings: Converging evidence from event-related potentials. Journal of Experimental Psychology: Human Perception \& Performance, 34, 509-530.

Maljkovic, V., \& Nakayama, K. (1994). Priming of pop-out: I. Role of features. Memory \& Cognition, 22, 657-672.

MeEter, M., \& Olivers, C. N. L. (2006). Intertrial priming stemming from ambiguity: A new account of priming in visual search. Visual Cognition, 13, 202-222.

Müller, H. J., Geyer, T., Zehetleitner, M., \& Krummenacher, J. (2009). Attentional capture by salient color singleton distractors is modulated by top-down dimensional set. Journal of Experimental Psychology: Human Perception \& Performance, 35, 1-16.

Müller, H. J., Heller, D., \& Ziegler, J. (1995). Visual search for singleton feature targets within and across feature dimensions. Perception \& Psychophysics, 57, 1-17.

Olivers, C. N. L., \& Humphreys, G. W. (2003). Attentional guidance by salient feature singletons depends on intertrial contingencies. Journal of Experimental Psychology: Human Perception \& Performance, 29, 650-657.

Olivers, C. N. L., \& Meeter, M. (2006). On the dissociation between compound and present/absent tasks in visual search: Intertrial priming is ambiguity-driven. Visual Cognition, 13, 202-222.

Peterson, L. R., \& Peterson, M. J. (1959). Short-term retention of individual verbal items. Journal of Experimental Psychology, 58, 193-198.
Pinto, Y., Olivers, C. N. L., \& Theeuwes, J. (2005). Target uncertainty does not lead to more distraction by singletons: Intertrial priming does. Perception \& Psychophysics, 67, 1354-1361.

Rauschenberger, R. (2003). Attentional capture by auto- and allocues. Psychonomic Bulletin \& Review, 10, 814-842.

SchreiJ, D., Owens, C., \& Theeuwes, J. (2008). Abrupt onsets capture attention independent of top-down control settings. Perception \& Psychophysics, 70, 208-218.

Theeuwes, J. (1990). Perceptual selectivity is task-dependent: Evidence from selective search. Acta Psychologica, 74, 81-99.

Theeuwes, J. (1991). Cross-dimensional perceptual selectivity. Perception \& Psychophysics, 50, 184-193.

Theeuwes, J. (1992). Perceptual selectivity for color and form. Perception \& Psychophysics, 51, 599-606.

Theeuwes, J. (1994). Stimulus-driven capture and attentional set: Selective search for color and visual abrupt onsets. Journal of Experimental Psychology: Human Perception \& Performance, 20, 799-806.

TheEuwes, J. (1995). Temporal and spatial characteristics of preattentive and attentive processing. Visual Cognition, 2, 221-233.

THEEUwes, J. (2004). Top-down search strategies cannot override attentional capture. Psychonomic Bulletin \& Review, 11, 65-70.

Theeuwes, J., Atchley, P., \& Kramer, A. F. (2000). On the time course of top-down and bottom-up control of visual attention. In S. Monsell \& J. Driver (Eds.), Attention and performance XVIII: Control of cognitive processes (pp. 105-124). Cambridge, MA: MIT Press.

Theeuwes, J., \& Godisn, R. (2002). Irrelevant singletons capture attention: Evidence from inhibition of return. Perception \& Psychophysics, 64, 764-770.

Theeuwes, J., Kramer, A. F., Hahn, S., \& Irwin, D. E. (1998). Our eyes do not always go where we want them to go: Capture of eyes by new objects. Psychological Science, 9, 379-385.

Theeuwes, J., Kramer, A. F., Hahn, S., Irwin, D. E., \& Zelinsky, G. J. (1999). Influence of attentional capture on oculomotor control. Journal of Experimental Psychology: Human Perception \& Performance, 25, 1595-1608.

Theeuwes, J., Reimann, B., \& Mortier, K. (2006). Visual search for featural singletons: No top-down modulation, only bottom-up priming. Visual Cognition, 14, 466-489.

Theeuwes, J., \& VAN DeR BurG, E. (2007). The role of spatial and nonspatial information in visual selection. Journal of Experimental Psychology: Human Perception \& Performance, 33, 1335-1351.

Theeuwes, J., \& VAN Der Burg, E. (2008). The role of cueing in attentional capture. Visual Cognition, 16, 232-247.

Theeuwes, J., VAN Der Burg, E., \& Belopolsky, A. (2008). Detecting the presence of a singleton involves focal attention. Psychonomic Bulletin \& Review, 15, 555-560.

Wolfe, J. M., Butcher, S. J., Lee, C., \& Hyle, M. (2003). Changing your mind: On the contributions of top-down and bottom-up guidance in visual search for feature singletons. Journal of Experimental Psychology: Human Perception \& Performance, 29, 483-502.

Yantis, S., \& Jonides, J. (1984). Abrupt visual onsets and selective attention: Evidence from visual search. Journal of Experimental Psychology: Human Perception \& Performance, 10, 601-621.

\section{NOTE}

1. Note that when the participants were set for WHITE, disengagement of attention from the color cue location might have sped up because the red cue was accompanied by white distractor cues. However, spatially selective suppression was also observed for the onset cues when the participants were set for RED, suggesting that the presence of distractors is not necessary.

(Manuscript received June 30, 2009; revision accepted for publication October 10, 2009.) 